\title{
Irreversible effects of memory
}

\author{
A. Puglisi ${ }^{1,2}$ and D. Villamaina ${ }^{1,2}$ \\ CNR-INFM SMC and ISC - p.le A. Moro 2, 00185, Roma, Italy \\ Dipartimento di Fisica, Università Sapienza, p.le A. Moro 2, 00185, Roma, Italy
}

PACS 05.40.-a - Fluctuation phenomena, random processes, noise, and Brownian motion PACS 05.70.Ln - Nonequilibrium and irreversible thermodynamics

\begin{abstract}
The steady state of a Langevin equation with short ranged memory and coloured noise is analyzed. When the fluctuation-dissipation theorem of second kind is not satisfied, the dynamics is irreversible, i.e. detailed balance is violated. We show that the entropy production rate for this system should include the power injected by "memory forces". With this additional contribution, the Fluctuation Relation is fairly verified in simulations. Both dynamics with inertia and overdamped dynamics yield the same expression for this additional power. The role of "memory forces" within the fluctuation-dissipation relation of first kind is also discussed.
\end{abstract}

Introduction. - Irreversibility of path probabilities accompanies the lack of thermodynamic equilibrium. This fact, which is obvious in relaxing systems, can also be shown in many models of statistically steady states. In the latter case there is the evidence for a general connection between irreversibility and power dissipated by non-conservative forces. A paradigm of this situation is a particle performing Brownian motion under the action of conservative and non-conservative forces:

$$
\dot{\mathbf{v}}=-\gamma \mathbf{v}-\nabla U(\mathbf{x})+\mathbf{F}_{n c}[\mathbf{x}(t)]+\boldsymbol{\eta},
$$

with $\boldsymbol{\eta}$ a Gaussian white noise, $\langle\boldsymbol{\eta}\rangle=0$ and $\left\langle\eta_{i}(t) \eta_{j}\left(t^{\prime}\right)\right\rangle=$ ${ }_{2} \gamma T \delta_{i j} \delta\left(t-t^{\prime}\right)$, where $i, j$ are component indices. It is easily shown that

$$
W_{t}=\log \frac{P\left(\{\mathbf{v}(s)\}_{0}^{t}\right)}{P\left(\{\mathcal{I} \mathbf{v}(s)\}_{0}^{t}\right)} \approx \frac{1}{T} \int_{0}^{t} \mathbf{F}_{n c}[\mathbf{x}(s)] \cdot \mathbf{v}(s) d s .
$$

where $\{\mathcal{I} \mathbf{v}(s)\}_{0}^{t}$ is the time-reversed trajectory. $W_{t}$ is usually called "produced entropy" [1-3], and in some deterministic models with certain assumptions it is equivalent, neglecting finite differences, to the time-integral of the phase space contraction rate $[4-6]$.

A phenomenon which may be enhanced in statistically steady states under the effect of non-conservative forces and/or multiple baths is that of correlations among degrees of freedom: if one insists on a reduced description of the system, such as the motion of a tracer particle, these correlations must be reintroduced as retarded feedback, or memory, accompanied by colored noise:

$$
\begin{aligned}
-\gamma \mathbf{v} & \rightarrow-\int_{-\infty}^{t} \gamma\left(t-t^{\prime}\right) \mathbf{v}\left(t^{\prime}\right) d t^{\prime} \\
\left\langle\eta_{i}(t) \eta_{j}\left(t^{\prime}\right)\right\rangle & \rightarrow M_{i j}\left(t-t^{\prime}\right) .
\end{aligned}
$$

We stress the fact that memory acts in equilibrated systems too, as extensively discussed in classical references [7], where the condition for thermal equilibrium, in the absence of non-conservative forces, is given by the fluctuation-dissipation relation of the second kind

$$
M_{i j}\left(t-t^{\prime}\right)=T \delta_{i j} \gamma\left(\left|t-t^{\prime}\right|\right),
$$

which guarantees: (a) canonical distribution, (b) equipartition between the particle and the surrounding fluid, (c) the validity of the fluctuation-dissipation relation (FDR) of the first kind $[8]$

$$
\frac{\overline{\delta v(t)}}{\delta v(0)}=\frac{1}{T}\langle v(t) v(0)\rangle
$$

Violations of Eq. (6) or (5) are associated with lack of equilibrium, i.e. presence of more than one thermostat and/or of non-conservative external forces. This is, for instance, the case of a granular liquid, that is a gas of inelastic hard particles coupled to an external thermostat and at a packing fraction between $20 \%$ and $50 \%$ : in this system it appears that particles are correlated among each

\footnotetext{
${ }^{1}$ where $\overline{ } \cdot$ and $\langle\cdot\rangle$ denote perturbed and unperturbed averages, respectively.
} 
others and this is reflected in a non-Markovian dynamics for a tracer, together with a violation of the Einstein relation [9-11]. A similar case is realized in a molecular liquid coupled to different thermostats, where the motion of a massive tracer is described by a Langevin equation with (short ranged) memory, and Eq. (5) is not satisfied [12].

Entropy production for Langevin models with shortranged memory has been recently studied in [13-16]. In the first three papers [13-15], the steady state is treated under the assumption of validity of (5), called "equilibrium" bath, so that the irreversibility takes again the form of work done by external non-conservative forces, similar to Eq. (2). In [14] a non-transparent general formula, for the case where (5) is violated, is also given, involving a triple time-convolution between forces, memory $\gamma(t)$ and the inverse of noise color $M(t)$. In [16], irreversibility of paths is given as a convolution of power dissipated by all (non-conservative and conservative) forces with a timedependent "effective temperature" $T_{e f f}(t)$ which characterizes the violation of (5). In the formulation of [16], the entropy production vanishes if both anharmonic potential and non-conservative external forces are absent.

In this letter we show, for rapidly decaying memory kernels with different time-scales, within a class of possible violations of Eq. (5), a simple formula, Eq. (35), for the entropy production: in our formula memory appears as a force performing work on the system, if and only if relation (5) is not satisfied. The fact that memory alone produces entropy has not been explicitly discussed in other references: this discrepancy is likely to be due to a different definition for entropy production. We consider the one given by Lebowitz and Spohn [2], which is related to the time-derivative of the Gibbs Entropy and which is properly derived if the dynamics is Markovian, i.e. all degrees of freedom required to determine the future are known in the present. To this purpose we consider a class of nonMarkovian dynamics which can be mapped to Markovian systems by introducing auxiliary degrees of freedom. An analogous approach is adopted in [11] in order to give an interpretation of "violations" of FDR.

It could be argued that our formula includes contributions to the entropy production coming from "internal forces", which is not physical in a steady state. On the contrary, we stress the general fact that memory cannot exist for an isolated particle: stated differently, the effect of past history influences the present only if other degrees of freedom (left out from the description) "remind" it to the particle. Recollisions in dense fluids constitute a typical mechanism [17]. Therefore memory should be seen as an "external" but hidden force. In the models considered here, Eqs. (24) and (39), this role is made explicit when the auxiliary variables are introduced. We stress the fact that, since only steady states are investigated here, cases with long-range memory are not analyzed [18].

The plan of the paper is the following: we first discuss our general formula, and give details for the inertial and overdamped dynamics of a tracer, showing that the
Fluctuation Relation [1,2] is verified for entropy production when the memory contribution is taken into account; then we analyze the connection between memory forces and the violation of FDR, previously studied in [11]; finally we draw conclusions and perspectives.

Irreversibility of multivariate paths. - Let us first discuss a non-multiplicative multivariate Langevin equation with $N$ degrees of freedom and without memory:

$$
\dot{X}_{i}=D_{i}(\mathbf{X})+g_{i j} \xi_{j}(t),
$$

with $i \in[0, N-1], \xi_{i}(t)$ is a Gaussian process with $\left\langle\xi_{i}(t)\right\rangle=0$ and $\left\langle\xi_{i}(t) \xi_{i}\left(t^{\prime}\right)\right\rangle=2 \delta_{i j} \delta\left(t-t^{\prime}\right)$. The probability distribution $f_{t}(\mathbf{X})$ satisfies

$$
\frac{\partial f_{t}(\mathbf{X})}{\partial t}=-\sum_{i} \frac{\partial S_{i}(\mathbf{X})}{\partial X_{i}}
$$

with the probability current defined by

$$
S_{i}(\mathbf{X})=D_{i}(\mathbf{X}) f_{t}(\mathbf{X})-\sum_{j} \frac{\partial}{\partial X_{j}} D_{i j} f_{t}(\mathbf{X})
$$

where $D_{i j}=\sum_{k} g_{i k} g_{j k}$ is symmetric by construction.

Variables $X_{i}$ are assumed to have a well-defined parity $\epsilon_{i}= \pm 1$, with respect to time-reversal. This leads to recognize reversible and irreversible parts of the drift:

$$
D_{i}(\mathbf{X})=D_{i}^{r e v}(\mathbf{X})+D_{i}^{i r}(\mathbf{X})
$$

with

$$
\begin{aligned}
D_{i}^{r e v}(\mathbf{X}) & =\frac{1}{2}\left[D_{i}(\mathbf{X})-\epsilon_{i} D_{i}(\epsilon \mathbf{X})\right]=-\epsilon_{i} D_{i}^{r e v}(\epsilon \mathbf{X}) \\
D_{i}^{i r}(\mathbf{X}) & =\frac{1}{2}\left[D_{i}(\mathbf{X})+\epsilon_{i} D_{i}(\epsilon \mathbf{X})\right]=\epsilon_{i} D_{i}^{i r}(\epsilon \mathbf{X})
\end{aligned}
$$

having defined $\epsilon \mathbf{X}=\left(\epsilon_{0} X_{0}, \epsilon_{1} X_{1}, \ldots \epsilon_{N-1} X_{N-1}\right)$. This decomposition can be extended to the probability current:

$$
S_{i}^{r e v}=f_{t} D_{i}^{r e v} \quad S_{i}^{i r}=S_{i}-S_{i}^{r e v}=f_{t} D_{i}^{i r}-\sum_{j} D_{i j} \frac{\partial f_{t}}{\partial X_{j}} .
$$

Following classical references $[19,20]$, the expression for conditional path probability of trajectory $\{\mathbf{X}(s)\}_{0}^{t}$, i. 2

$$
\begin{aligned}
\log P\left(\{\mathbf{X}(s)\}_{0}^{t}\right)=-\frac{1}{4} \sum_{j k} & \int_{0}^{t} d s D_{j k}^{-1}\left\{\dot{X}_{j}(s)-D_{j}[\mathbf{X}(s)]\right\} \\
& \times\left\{\dot{X}_{k}(s)-D_{k}[\mathbf{X}(s)]\right\}, \quad(14)
\end{aligned}
$$

where we have assumed that $D_{i j}^{-1}$ exists.

By using Eq. (10) and a few passages we get

$$
\begin{gathered}
W_{t}=\log \frac{P\left(\{\mathbf{X}(s)\}_{0}^{t}\right)}{P\left(\{\mathcal{I} \mathbf{X}(s)\}_{0}^{t}\right)}=-\frac{1}{2} \int_{0}^{t} d s D_{j k}^{-1} \times \\
\left\{a_{j k}^{-}\left[\dot{X}_{j} \dot{X}_{k}+D_{j}^{i r} D_{k}^{i r}+D_{j}^{r e v} D_{k}^{r e v}-2 \dot{X}_{j} D_{k}^{r e v}\right]\right. \\
\left.-2 a_{j k}^{+}\left[D_{j}^{i r} \dot{X}_{k}-D_{j}^{i r} D_{k}^{r e v}\right]\right\}
\end{gathered}
$$

\footnotetext{
${ }^{2}$ We use the Ito convention for stochastic integrals. Note that, with this convention, the Jacobian in the path probability is 1 [21].
} 
where we have introduced the following definitions:

$$
a_{j k}^{-}=\frac{1-\epsilon_{j} \epsilon_{k}}{2}, \quad a_{j k}^{+}=\frac{1+\epsilon_{j} \epsilon_{k}}{2} .
$$

Eq. (15) is strongly simplified in the case of a diagona 3 diffusion matrix $D_{i j}$, obtaining:

$$
W_{t}=\sum_{k} D_{k k}^{-1} \int_{0}^{t} d s D_{k}^{i r}\left[\dot{X}_{k}-D_{k}^{r e v}\right] .
$$

We are not aware of any previous derivation of this formula in the literature. It is not difficult to generalize it to multiplicative processes (i.e. $D_{i j}$ dependent upon $\mathbf{X}$ ).

We now recall that, in order to obtain the complete path probability in the steady state, one has to multiply $P\left(\{\mathbf{X}(s)\}_{0}^{t}\right)$ by $f(\mathbf{X}(0))$, where $f=\lim _{t \rightarrow+\infty} f_{t}$ is the stationary probability distribution. It is therefore possible to compute a different quantity

$$
\begin{array}{r}
W_{t}^{\prime}=\log \frac{f[\mathbf{X}(0)] P\left(\{\mathbf{X}(s)\}_{0}^{t}\right)}{f[\epsilon \mathbf{X}(t)] P\left(\{\mathcal{I} \mathbf{X}(s)\}_{0}^{t}\right)}=W_{t}+b_{t} \\
b_{t}=\log \{f[\mathbf{X}(0)]\}-\log \{f[\epsilon \mathbf{X}(t)]\} .
\end{array}
$$

The term $b_{t}$ is usually known as "border term" [22].

It is interesting also to define "entropy production rates" $\sigma$ and $\sigma^{\prime}$, such that $W_{t}=\int_{0}^{t} \sigma(s) d s$ and $W_{t}^{\prime}=\int_{0}^{t} \sigma^{\prime}(s) d s$. The following decomposition can be done:

$$
\begin{aligned}
\sigma & =\frac{d}{d t}(\log f)+\sigma_{1}+\sigma_{2}, \quad \sigma^{\prime}=\sigma_{1}+\sigma_{2} \\
\sigma_{1} & =\sum_{i} \dot{X}_{i}\left(-\frac{\partial \log f}{\partial X_{i}}+D_{i i}^{-1} D_{i}^{i r}\right) \\
\sigma_{2} & =-\sum_{i} D_{i i}^{-1} D_{i}^{i r} D_{i}^{r e v} .
\end{aligned}
$$

This decomposition will be particularly useful in the last section, when discussing the connection with the FDR.

The condition of detailed balance is equivalent to $\sigma^{\prime} \equiv$ 0 for all trajectories in the steady state. When detailed balance does not hold, it can be shown that

$$
\log \frac{\mathrm{p}\left(W_{t}^{\prime}=x\right)}{\mathrm{p}\left(W_{t}^{\prime}=-x\right)}=x,
$$

where $p\left(W_{t}^{\prime}=x\right)$ is the probability in the steady state. Eq. (23) is the finite-time Fluctuation Relation (FR). In general, excluding cases discussed in the literature [22-25], for large times $t$ one has $W_{t} \approx W_{t}^{\prime}$ and relation (23) is also satisfied by $W_{t}[1-3]$.

Memory through auxiliary variables. - The first class of generalized Langevin equations with memory we are interested in, concerns inertial dynamics, i.e. we consider the following equation of motion (we restrict ourselves to the one-dimensional problem, without loss of generality):

$$
\left\{\begin{aligned}
\dot{x} & =v \\
\dot{v} & =F(x)-\int_{-\infty}^{t} \gamma\left(t-t^{\prime}\right) v\left(t^{\prime}\right) d t^{\prime}+\eta(t)
\end{aligned}\right.
$$

\footnotetext{
${ }^{3}$ for the models presented here, this is the only relevant case.
}

with

$$
\begin{aligned}
\gamma(t) & =2 \gamma_{0} \delta(t)+\sum_{i=1}^{M} \frac{\gamma_{i}}{\tau_{i}} e^{-\frac{t}{\tau_{i}}} \quad\langle\eta(t)\rangle=0 \\
\left\langle\eta(t) \eta\left(t^{\prime}\right)\right\rangle & =2 T_{0} \gamma_{0} \delta\left(t-t^{\prime}\right)+\sum_{i=1}^{M} T_{i} \frac{\gamma_{i}}{\tau_{i}} e^{-\frac{\left|t-t^{\prime}\right|}{\tau_{i}}} .
\end{aligned}
$$

and where $F$ is a generic drift term which can take the form of a sum of conservative and non-conservative forces, i.e. $F(x) \equiv-\frac{d U_{0}(x)}{d x}+F_{n c}(x)$. When $T_{i}=T_{0}$ for all $i$, the FDR of the second kind, Eq. (5), holds. This model has several applications: among others, it has been proposed in [12] for weakly driven glassy systems; the dynamics of a tracer particle in moderately dense fluidized granular media, including its linear response properties, are consistent with this model [9], and, recently, the noise in feedback cooled oscillator for gravitational wave detectors [26] has been characterized in a similar fashion [27] [4

It is useful to map Eq. (24) into Eq. (7), where all noises are uncorrelated, identifying $N=M+2$ and $X_{0} \equiv v$, $X_{N-1} \equiv x$, while $X_{i} \equiv v_{i}(i \in[1, M])$ are auxiliary variables necessary to take into account memory, for instance they can be defined as

$$
v_{i}(t)=\sqrt{\frac{\gamma_{i}}{\tau_{i}}} \int_{-\infty}^{t} e^{-\frac{t-t^{\prime}}{\tau_{i}}}\left(v\left(t^{\prime}\right)+\sqrt{\frac{T_{i}}{\gamma_{i}}} \xi_{i}\left(t^{\prime}\right)\right) d t^{\prime} .
$$

With this choice for the auxiliary variables, it is easy to verify that the drifts in Eq. (77) are

$$
\begin{aligned}
D_{0} & =F(x)-\gamma_{0} v-\sum_{i=1}^{M} \sqrt{\frac{\gamma_{i}}{\tau_{i}}} v_{i} \\
D_{i} & =\sqrt{\frac{\gamma_{i}}{\tau_{i}}} v-\frac{1}{\tau_{i}} v_{i} \quad(i \in[1, M]) \\
D_{N-1} & =v,
\end{aligned}
$$

while the diagonal diffusion matrix reads

$$
D_{00}=\gamma_{0} T_{0} \quad D_{i i}=\frac{T_{i}}{\tau_{i}}
$$

Summarizing, the system with memory is recast into a system of (linearly) coupled Langevin equations where all noises are uncorrelated. Auxiliary variables $v_{i}(i \in[1, M])$ are even under time-reversal, i.e. $\epsilon_{i}=1$ for $i>0$ : this can be understood, for instance, requiring the validity of detail balance in the equilibrium case $T_{i}=T_{0}$ for all $i$.

Then we obtain

$$
\begin{aligned}
D_{0}^{r e v} & =F-\sum_{i=1}^{M} \sqrt{\frac{\gamma_{i}}{\tau_{i}}} v_{i}, \quad D_{0}^{i r}=-\gamma_{0} v \\
D_{i}^{r e v} & =\sqrt{\frac{\gamma_{i}}{\tau_{i}}} v, \quad D_{i}^{i r}=-\frac{v_{i}}{\tau_{i}} \quad(i \in[1, M]) \\
D_{N-1}^{r e v} & =v, \quad D_{N-1}^{i r}=0 .
\end{aligned}
$$

\footnotetext{
${ }^{4}$ Note that the pairing of equal characteristic times for the exponentials in 25) and 26 is not so restrictive: indeed, case $T_{i}=0$ or case $\gamma_{i} \rightarrow 0, T_{i} \rightarrow \infty$ with finite $\gamma_{i} T_{i}$, for some $i$, can be easily worked out and make no exception to the following analysis.
} 
When computing Eq. (17), it is crucial to note that $D_{i j}$ is not positive definite and cannot be inverted. Anyway, as noted by Machlup and Onsager [28], the last row and column of $D_{i j}$ (those associated to variable $x$, which has not explicit noise dependence) can be dropped out for the purpose of computing path probabilities. With this observation, formula (17) can be used, leading to

$$
\begin{aligned}
W_{t} & =-\sum_{i=0}^{M} \frac{\delta\left(v_{i}^{2}\right)}{2 T_{i}}-\frac{\delta U_{0}}{T_{0}}+ \\
& +\int_{0}^{t} \frac{1}{T_{0}}\left(F_{n c}[x(s)]+\sum_{i} F_{i}\left[v_{i}(s)\right]\right) v(s) d s \\
F_{i} & =-\sqrt{\frac{\gamma_{i}}{\tau_{i}}}\left(1-\frac{T_{0}}{T_{i}}\right) v_{i}(s) .
\end{aligned}
$$

As usual, exact differences appear, denoted as $\delta(g) \equiv$ $g(t)-g(0)$. The non-trivial part of $W_{t}$ is the time-integral on the r.h.s. of (35), which does not reduce to exact differences: it is equivalent to the work done by the usual non-conservative external force $F_{n c}(x)$ and by new forces $F_{i}$ expressed in Eq. (36). It can be verified that memory forces do not depend on the definition of auxiliary variables, Eq. (27), as expected. The additional work done by forces $F_{i}$ is due to feedback of past history on the particle velocity and it is interesting to discover its effect on irreversibility. From formula (35) it is also evident that force $F_{i}$ vanishes if $T_{i}=T_{0}$. If $T_{i}=T_{0}$ for all $i$, then the second kind FDR, Eq. (5), holds, and memory does not contribute to $W_{t}$.

We conclude this section, evaluating the so-called finite time (or transient) contribution $b_{t}$ which must be added in order to verify the FR at short times [22], in the particular case $M=1$ (only one auxiliary variable). We consider a setup where there is no external force, $F=0$, apart from two reflecting walls confining the particle to have a uniform spatial distribution in between, making irrelevant the contribution of $x$ to the stationary probability density. Considering only variables $\mathbf{v}=\left(v_{0} \equiv v, v_{1}\right)$, one has:

$$
f(\mathbf{v}) \propto \exp \left(-\frac{1}{2} \sum_{i j} \Sigma_{i j}^{-1} v_{i} v_{j}\right),
$$

where the inverse covariance matrix elements read $\Sigma_{00}^{-1}=$ $\frac{1}{T_{0}}\left(1+\frac{T^{*}}{Q} \Delta T\right), \Sigma_{11}^{-1}=\frac{1}{T_{1}}\left(1-\frac{\gamma_{0} \tau_{1} T^{*}}{Q} \Delta T\right)$ and $\Sigma_{01}^{-1}=$ $-\frac{\gamma_{0}}{\gamma_{1}} \sqrt{\gamma_{1} \tau_{1}} \frac{1+\gamma_{0} \tau_{1}}{Q} \Delta T$, with $\Delta T=T_{0}-T_{1}, T^{*}=\gamma_{0} \tau_{1} T_{0}+T_{1}$ and $Q=\left(T^{*}\right)^{2}+\frac{\gamma_{0}}{\gamma_{1}} T_{0} T_{1}\left(1+\gamma_{0} \tau_{1}\right)^{2}$. In summary:

$$
b_{t}=\Sigma_{00}^{-1} \frac{\delta v^{2}}{2}+\Sigma_{11}^{-1} \frac{\delta v_{1}^{2}}{2}-\Sigma_{01}^{-1}\left[v_{1}(0) v(0)+v_{1}(t) v(t)\right]
$$

Let us note that, when $T_{0}=T_{1}$ (validity of the FDR of the second kind), the exact difference (first term) of $W_{t}$ exactly cancels $b_{t}$ : in particular, if external non-conservative forces are absent, it appears that $W_{t}^{\prime}=0$, i.e. detailed balance is satisfied.

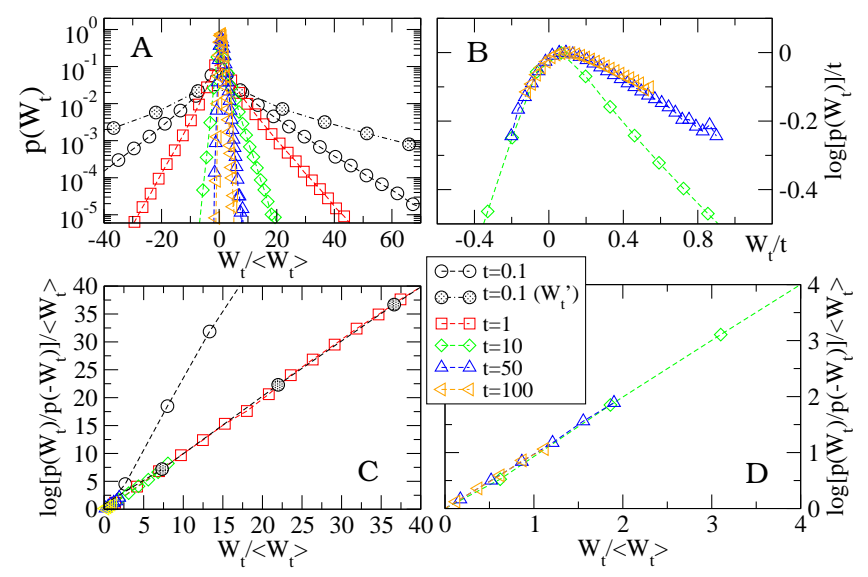

Fig. 1: A: pdf of $W_{t}$ for the inertial Langevin equation 24 with simple exponential memory, i.e. $M=1, T_{0}=0.6, T_{1}=3$, $\gamma_{0}=10, \tau_{1}=10, \gamma_{1}=5$, and $F=0$, for different times $t$ of integration. B: at large times, $\log p\left(W_{t} / t\right) / t$ converges to a time-independent function: the large deviation rate. CD: check of the Fluctuation Relation (FR), Eq. (23) which is verified for all data aligned along the bisector. At small time (empty circles), where the FR does not hold, Eq. (23) is verified for the pdf of $W_{t}^{\prime}$ (gray circles). Note that the only source of entropy, for this example, is memory.

In Figure 1 we show the probability density function (pdf) $p\left(W_{t}\right)$, in the steady state, obtained numerically by integrating Eq. (24), for different choices of times. In the same figure we also show the validity of Eq. (23) for $W_{t}$ at large times and $W_{t}^{\prime}$ at any time (see the difference between empty and gray circles), as well as the asymptotic convergence to the large deviation rate function.

The overdamped limit. - In the overdamped limit, the role of main variable is played by the tracer position $x$, while velocity $v$ is neglected. Even if the dynamics is changed, and drift terms have different symmetries with respect to time-reversal, the final result appears identical, making robust our observation.

The overdamped limit of Eq. (24) is a generalized Langevin equation with memory for the tracer position:

$$
\begin{aligned}
\gamma_{0} \dot{x}=-\frac{d U_{0}(x)}{d x} & +F_{n c}(x)+ \\
& -\sum_{i=1}^{M} \frac{\gamma_{i}}{\tau_{i}} \int_{-\infty}^{t} e^{-\frac{t-t^{\prime}}{\tau_{i}}} \dot{x}\left(t^{\prime}\right) d t^{\prime}+\eta(t)
\end{aligned}
$$

with the same properties as in Eq. (26) for the noise $\eta(t)$. With a partial integration it is possible to cast Eq. (39) into

$$
\begin{aligned}
\gamma_{0} \dot{x}=-\frac{d U_{0}(x)}{d x} & +F_{n c}(x)-\sum_{i=1}^{M} \frac{\gamma_{i}}{\tau_{i}} x+ \\
& +\sum_{i=1}^{M} \frac{\gamma_{i}}{\tau_{i}^{2}} \int_{-\infty}^{t} e^{-\frac{t-t^{\prime}}{\tau_{i}}} x\left(t^{\prime}\right) d t^{\prime}+\eta(t) .
\end{aligned}
$$


We use now the following auxiliary variables, with $i \in$ $[1, M]$ :

$$
x_{i}(t)=\frac{1}{\tau_{i}} \int_{-\infty}^{t} e^{-\frac{t-t^{\prime}}{\tau_{i}}}\left(x\left(t^{\prime}\right)+\tau_{i} \sqrt{\frac{T_{i}}{\gamma_{i}}} \xi_{i}\left(t^{\prime}\right)\right) d t^{\prime},
$$

obtaining an equivalence with a system of Langevin equations without memory of the form (7), with $N=M+1$ (identifying $X_{0} \equiv x$ and $X_{i} \equiv x_{i}$ ), and with

$$
\begin{aligned}
\gamma_{0} D_{0} & =-\frac{d U_{0}(x)}{d x}+F_{n c}(x)-\sum_{i=1}^{M} \frac{\gamma_{i}}{\tau_{i}}\left(x-x_{i}\right) \\
D_{i} & =\frac{1}{\tau_{i}}\left(x-x_{i}\right) \quad(i>0), \quad D_{i j}=\delta_{i j} \frac{T_{i}}{\gamma_{i}} .
\end{aligned}
$$

Following the same procedure of identification of irreversible and reversible parts of drifts, and recognizing again that memory auxiliary variables have parity $\epsilon_{i}=1$, we obtain

$$
D_{i}^{r e v}=0, \quad D_{i}^{i r}=D_{i} .
$$

This leads to identifying, for the entropy production, the following expression, after suitable partial integrations:

$$
\begin{aligned}
W_{t}=-\sum_{i=0}^{M} \frac{\delta U_{i}}{T_{i}}+\int \frac{1}{T_{0}}\{ & F_{n c}[x(s)]+ \\
& \left.+\sum_{i=1}^{M} F_{i}[x(s)]\right\} \dot{x}(s) d s
\end{aligned}
$$

where

$$
\begin{aligned}
U_{i}(t) & =\frac{\gamma_{i}}{2 \tau_{i}}\left[x(t)-x_{i}(t)\right]^{2} \quad(i>0) \\
F_{i} & =\frac{\gamma_{i}}{\tau_{i}}\left(1-\frac{T_{0}}{T_{i}}\right)\left[x_{i}(s)-x(s)\right] .
\end{aligned}
$$

As seen, also for the overdamped case, entropy production is equivalent to the work done by the external nonconservative force plus non-conservative forces $F_{i}(s)$ due to memory. It is easy to verify, through a partial integration and the comparison between Eq. (27) and Eq. (41), that

$$
\frac{\gamma_{i}}{\tau_{i}}\left[x_{i}(t)-x(t)\right]=-\sqrt{\frac{\gamma_{i}}{\tau_{i}}} v_{i}(t),
$$

and therefore the forces in Eq. (47) are exactly equivalent to the forces in Eq. (36) for the inertial dynamics. Again we have verified, through numerical integration of Eq. (39), that the pdf of $W_{t}$ for large times reproduces the FR. For short times, the terms $b_{t}$ due to the steady state invariant measure at initial and final configurations must be added in order to recover the short-times kind of symmetry. The same consideration drawn for the inertial dynamics can be repeated here: when $T_{i}=T$, for all $i$, i.e. when the FDR of the second kind holds, then $F_{i}=0$ and the boundary term $b_{t}$ cancels out the exact differences in Eq. (45), so that, if $F_{n c}=0$, detailed balance holds.
In conclusion, the only difference between expression (35) and (45) for the entropy production, is given by exact differences: these differences, for large times, can be neglected and the two expressions become equivalent. This is coherent with the fact that the overdamped dynamics ignores the short time-scale corresponding to the relaxation of velocity.

Linear response. - The first-kind FDR for the overdamped model in (39) has been treated in several papers, see for instance $[12,16]$. More in general, it is known that a generalized FDR $[8,11,29-31]$ is satisfied if all variables $\mathbf{X} \equiv X_{0}, X_{1}, \ldots, X_{M}$ are taken into account with their steady state invariant measure $f(\mathbf{X})$, provided that it is smooth and non vanishing, and the system is mixing.

If an impulsive variation of coordinates $\left\{\delta X_{i}(0)\right\}$ at time 0 is considered, the generalized FDR for the response takes the form

$$
\begin{aligned}
R_{j i} \stackrel{\text { def }}{=} \frac{\overline{\delta X_{j}(t)}}{\delta X_{i}(0)}=\left\langle X_{j}(t) B_{i}(0)\right\rangle \\
B_{i}=-\frac{\partial \log f(\mathbf{X})}{\partial X_{i}}
\end{aligned}
$$

where we use - to mean non-equilibrium averages following the perturbation at time 0 , while $\langle\cdot\rangle$ represents an ensemble average, which (under ergodicity) is equivalent to averaging over a long trajectory in the stationary state. If the diffusion matrix, $D_{i j}$, is diagonal, one has [20]:

$$
\begin{aligned}
& B_{i}=B_{i}^{0}+B_{i}^{*} \\
& B_{i}^{0}=-D_{i i}^{-1} D_{i}^{i r}, \quad B_{i}^{*}=\frac{S_{i}^{i r}}{D_{i i} f} .
\end{aligned}
$$

When detailed balance is satisfied, which implies that $W_{t}=0$ on average and $W_{t}^{\prime}=0$ for each trajectory, one has $S_{i}^{i r}=0[20]$ and therefore

$$
R_{j i}=R_{j i}^{0} \stackrel{\text { def }}{=}\left\langle X_{j}(t) B_{i}^{0}(0)\right\rangle=-\left\langle X_{j}(t) \frac{D_{i}^{i r}(0)}{D_{i i}}\right\rangle .
$$

It is straightforward to verify that Eq. (53) takes standard equilibrium forms, e.g. it is equivalent to Eq. (6) for velocity, or more in general to Kubo relations [7,20], which depends upon the choice of system, perturbation and measured responses. As discussed in previous sections, for models (24) and (39), when external non-conservative forces are absent, detailed balance condition $S_{i}^{i r}=0$ corresponds to $T_{i}=T_{0}$ for all $i$, i.e. to the FDR of the second kind, Eq. (5).

On the contrary, when detailed balance is not satisfied, the response includes an additive contribution:

$$
R_{j i}=R_{j i}^{0}+\left\langle X_{j}(t) B_{i}^{*}(0)\right\rangle .
$$

Interestingly, comparison of Eqs. (13), (21) and (52) gives the following identification:

$$
\sigma_{1}=\sum_{i} \dot{X}_{i} B_{i}^{*}
$$


This relation illustrates the connection between the "violation" of Eq. (53) and a part of the entropy production, $\sigma_{1}$. Indeed, relation (55) becomes very useful in the overdamped dynamics, where $\sigma_{2}=0$ : in this case the generalized force $B_{i}^{*}$ which entirely contributes to the entropy production $\sigma^{\prime}=\sigma_{1}$, is the same force acting as "conjugate quantity" in the additional (non-equilibrium) terms of the linear response formula. In the case with inertia, relation (55) becomes less useful, since $\sigma_{1}$ only contains exact differential and the bulk contribution to the entropy production comes from $\sigma_{2}$.

We wish to point out that other approaches toward the connection between FDR and entropy production have also been discussed, from different points of view, in many recent works, e.g. [32-35]: for a detailed review, see also [36].

Conclusions. - Summarizing, we have discussed the role of memory in non-equilibrium steady states, merging two main observations: 1) a general formula for entropy production in multivariate memory-less Langevin processes and 2) the mapping between Langevin models with short-ranged memory toward memory-less Langevin models with auxiliary variables. The latter leads to identify memory as a non-conservative force. These forces cease to contribute to entropy production only under the validity of the FDR of the second kind, Eq. (5).

Interestingly, in the overdamped dynamics, these same forces contribute to the so-called "violations" of the FDR of the first kind, when detailed balance is not satisfied. Future work should include a generalization to Langevin equations with other forms of memory. Investigation of the case with inertia is also needed, to better explore the connections with the linear response theory.

\section{$* * *$}

The work of the authors is supported by the "GranularChaos" project, funded by the Italian MIUR under the FIRB-IDEAS grant number RBID08Z9JE. Both authors wish to thank P. De Gregorio, G. Gonnella, L. Rondoni, P. Visco and A. Vulpiani for useful discussions.

\section{REFERENCES}

[1] Kurchan J., J. Phys. A , 31 (1998) 3719.

[2] Lebowitz J. L. and Spohn H., J. Stat. Phys. , 95 (1999) 333.

[3] Seifert U., Phys. Rev. Lett. , 95 (2005) 040602.

[4] Evans D. J., Cohen E. G. D. and Morriss G. P., Phys. Rev. Lett. , 71 (1993) 2401.

[5] Evans D. J. and Searles D. J., Phys. Rev. E , 50 (1994) 1645.

[6] Gallavotti G. and Cohen E. G. D., J. Stat. Phys. , 80 (1995) 931.

[7] Kubo R., Toda M. and Hashitsume N., Statistical physics II: Nonequilibrium stastical mechanics (Springer) 1991.
[8] Marconi U. M. B., Puglisi A., Rondoni L. and VulpiANi A., Phys. Rep. , 461 (2008) 111.

[9] Puglisi A., Baldassarri A. and Vulpiani A., J. Stat. Mech. , (2007) P08016.

[10] Villamaina D., Puglisi A. and Vulpiani A., J. Stat. Mech. , (2008) L10001.

[11] Villamaina D., Baldassarri A., Puglisi A. and Vulpiani A., J. Stat. Mech. , (2009) P07024.

[12] Cugliandolo L. F. and Kurchan J., J Phys Soc Jpn, 69 (2000) 247.

[13] Speck T. and Seifert U., J. Stat. Mech. , (2007) L09002.

[14] Ohkuma T. and Онта T., J. Stat. Mech. , (2007) $\mathrm{P} 10010$

[15] Mai T. and Dhar A., Phys. Rev. E , 75 (2007) 061101.

[16] Zamponi F., Bonetto F., Cugliandolo L. F. and Kurchan J., J. Stat. Mech. , (2005) P09013.

[17] McLennan J. A., Introduction to Nonequilibrium Statistical Mechanics (Prentice-Hall) 1989.

[18] Harris R. J. and Touchette H., J. Phys. A: Math. Theor., 42 (2009) 342001.

[19] Onsager L. and Machlup S., Phys. Rev. , 91 (1953) 1505.

[20] Risken H., The Fokker-Planck equation: Methods of solution and applications (Springer- Verlag, Berlin) 1989.

[21] HängGi P., Path integral solution for nonlinear Generalized Langevin equations in proc. of Path Integrals for meV to MeV: Tutzing '92, edited by Grabert H., Inomata A., Schulman L. and Weiss U., (World Scientific) 1993 p. 289.

[22] Puglisi A., Rondoni L. and Vulpiani A., J. Stat. Mech. , (2006) P08010.

[23] van Zon R. and Cohen E. G. D., Phys. Rev. Lett. , 91 (2003) 110601.

[24] Evans D., Searles D. and Rondoni L., Phys. Rev. E, 71 (2005) 056120.

[25] Bonetto F., Gallavotti G., Giuliani A. and ZamPONi F., J. Stat. Phys., 123 (2006) 39.

[26] Bonaldi M. and Et AL., Phys. Rev. Lett. , 103 (2009) 010601.

[27] Gregorio P. D., Rondoni L., Bonaldi M. and Conti L., arXiv:090\%.4309 , (2009).

[28] Machlup S. and Onsager L., Phys. Rev. , 91 (1953) 1512.

[29] Agarwal G. S., Z. Physik, 252 (1972) 25.

[30] Deker U. and Hanke F., Phys. Rev. A , 11 (1975) 2043.

[31] Falcioni M., Isola S. and Vulpiani A., Physics Letters A , 144 (1990) 341.

[32] Hatano T. and Sasa S., Phys. Rev. Lett. , 86 (2001) 3463.

[33] Lippiello E., Corberi F. and Zannetti M., Phys. Rev. E, 71 (2005) 036104.

[34] Speck T. and Seifert U., Europhys. Lett. , 74 (2006) 391.

[35] Baiesi M., Maes C. and Wynants B., Phys. Rev. Lett. , 103 (2009) 010602.

[36] Speck T. and Seifert U., arXiv:0907.5478, (2009). 